\title{
Clinical research of effects of retaining the uterine blood supply hysterectomy on ovarian function
}

\author{
Yufei Cai ${ }^{1}$ and Hongxia Sun ${ }^{2, a}$ \\ ${ }^{1}$ Obstetrics and Gynecology Department,Affiliated Hospital of Beihua University \\ ${ }^{2}$ Pharmacological Department, Pharmacy, Beihua University jilin jilin 132011, China
}

\begin{abstract}
Objective To evaluate the effect of hysterectomy for reserving the uterine blood supply on ovarian endocrine function and on symptoms of menopausal transition. Methods Uterine benign lesions should be line the uterus times total resection in 100 patients were randomly divided into hysterectomy group of retaining uterus vascular supply group(research group, $\mathrm{n}=50$ ) and traditional total hysterectomy group (the control group, $\mathrm{n}=50$ ), comparing two groups in operation time, intraoperative bleeding, postoperative fever and residual polyp, blood tests were taken to check the serum sex hormone levels change and clinical observation for perimenopausal symptoms before and postoperative three months, six months, one year and two years at the same time respectively. Results There was no significant difference between two groups in operation time, intraoperative blood loss, postoperative fever and residual polyp $(\mathrm{P}>0.05)$. There was no significant difference among research group before and after operation in serum sex hormones $(\mathrm{P}>0.05)$, the symptoms of the menopausal transition hardly appear; postoperative FSH, LH increased significantly in control group $(\mathrm{P}<0.05)$, E2 decrease $(\mathrm{P}<0.05)$, perimenopausal symptoms appeared more often. Conclusion The effect of uterus hysterectomy for retaining vascular supply on ovarian endocrine function is less than the traditional total hysterectomy, this operation has a certain importance to preserve ovarian function and delay the occurrence of premature ovarian aging.
\end{abstract}

\section{Instruction}

The endocrine function of ovary can be affected by hysterectomy. In traditional hysterectomy, cutting off the ovarian branch of uterine blood vessels could reduce the ovarian blood supply by fifty to seventy percent, leading to ovarian follicle degeneration to reduce hormone production and ovarian function declined four to five years after hysterectomy, which increased the occurrence of complications such as peri-menopausal symptoms, osteoporosis, high blood lipids and coronary heart disease and so on. Subtotal hysterectomy for maintaining uterine vessels (STHMUV) is a kind of surgery that does not affect ovarian hormone secretion, which can ensure the blood supply to the ovaries after removal of uterus, STHMUV can delay the natural aging of the ovary. So that the ovary is still able to secrete estradiol and other hormones, which delayed the emergence of ovarian physiological decline [1].

\footnotetext{
${ }^{a}$ Corresponding authorsunhongxiaj1@126.com
} 


\section{Materials and methods}

\subsection{General information}

One hundreds cases of patients which had sign of subtotal resection of uterus (SROU), aging between thirty to forty-five years old, was selected from the Department of gynecology in the affiliated hospital of Beihua University during the period from December 2014 to October 2010. and were randomly divided into STHMUV group (research group, $n=50$, indicating $\mathrm{R}$ group) and traditional SROU group (control group, $\mathrm{n}=50$, indicating $\mathrm{C}$ group). There was no significant difference in age, types of diseases in the two groups $(\mathrm{P}>0.05)$.Cervix was examined and serum lipid levels were detected before operation. All women were admitted to hospital within three to five days after the last menstrual period. There was no significant difference in serum TC, HDI, TG and LDL in the two groups $(\mathrm{P}>0.05)$.

\subsection{Choice of operation mode}

The control group with reference to Li Guangyi, editor in chief of "Practical Gynecology laparoscopic surgery" for the traditional subtotal hysterectomy [10]. The study group adopted the reservation of the uterine vessels subtotal hysterectomy. The study group and the control group were the same, both of which were combined with the spinal epidural anesthesia. The oxytocin and saltwater were mixed to be injected into the uterus, its vasoconstrictive effects lead to the whole uterine tissue became pale due to ischemia, the remaining part of the liquid was dumped into the contralateral body layer during the surgery; cross-cutting and episiotomy surgery could be taken in lower abdomen, each layer was cut to be checked in order by open view according to its anatomical position, the uterus was maintained in a raised position during the operation, both sides of the uterine appendages and uterine blood vessels was clamped with tissue forceps. The position for uterine resection was about $1 \mathrm{~cm}$ interior to uterine horn, toward the direction of uterine isthmus, resection of uterine body by the shape of triangle. The lower position was close to the inside of uterus cervix, with about $2 \mathrm{~cm}$ thickness of both side of uterine body. The muscle and endometrial layers can be clearly seen at this point, if there was,such as the obvious hysteromyoma, which can be removed, with hemostatic forceps or electric hemostasis. The uterine wound was sutured by U-shaped, and check whether there is staxis or not meanwhile.

\subsection{Observation index}

Operation time, bleeding during operation, postoperative fever and residual polyps were compared between the two groups. Serum lipid levels were taken to detected three months, six months, one year and two years after operation and compare the serum lipid levels, hemodynamic of uterine artery and survey of sexual satisfaction in different time periods.

\subsection{Statistical methods}

SPSS 17.0 statistical software was used for statistical analysis, all data were expressed as mean \pm the standard deviation $(\bar{x} \pm$ s), single factor analysis of variance was used for comparison among groups, LSD-t test was used for comparison between two groups, the difference was statistically significant when $P<0.05$. 


\section{Results}

\subsection{Operation effects}

Operation time, intraoperative blood loss, postoperative body temperature and postoperative residual segment polyps were compared between two groups of, the results showed that there was no significant differences $(\mathrm{P}>0.05)$, see Table 1 .

Table 1. Comparison of two groups of patients with operation $(x \pm s)$

\begin{tabular}{|c|c|c|c|c|c|}
\hline Group & $n$ & Operation T/min & $\begin{array}{c}\text { Blood } \\
\text { loss } / \mathrm{ml}\end{array}$ & $\mathrm{BT} /{ }^{\circ} \mathrm{C}$ & $\begin{array}{c}\text { Residual polyps } \\
/ \%\end{array}$ \\
\hline Research(R) & 50 & $53.2 \pm 7.6$ & $90 \pm 12.6$ & $37 \pm 0.5$ & $0 / 0$ \\
\hline Control(C) & 50 & $55.1 \pm 8.3$ & $95 \pm 16.7$ & $37.5 \pm 0.2$ & $28 / 56$ \\
\hline
\end{tabular}

\subsection{Sexual hormones level comparision in serum}

Sexual hormone in serum before and after operation is shown in Tab 2

Table 2. The comparison results of serum sexual hormone levels before and after two groups operation $\left(\bar{x}_{ \pm \mathrm{s}}\right)$

\begin{tabular}{|c|c|c|c|c|c|c|}
\hline \multirow{2}{*}{ Group } & \multirow{2}{*}{$\mathrm{n}$} & \multicolumn{5}{|c|}{$\mathrm{FSH} / \mathrm{U} \cdot \mathrm{L}^{-1}$} \\
\hline & & before & $3 \mathrm{~m}$ after operation & $6 \mathrm{~m}$ after operation & 1y after operation & $2 y$ after operation \\
\hline Research & 50 & $17.65 \pm 1.02$ & $18.10 \pm 1.31$ & $18.67 \pm 1.74 *$ & $19.45 \pm 1.68^{*}$ & $20.17 \pm 1.25^{*}$ \\
\hline Control & 50 & $16.32 \pm 1.41$ & $16.05 \pm 1.06$ & $26.35 \pm 1.89^{\triangle}$ & $53.36 \pm 1.74^{\triangle}$ & $89.01 \pm 1.96^{\triangle}$ \\
\hline Group & $\mathrm{n}$ & \multicolumn{5}{|c|}{$\begin{array}{c}\mathrm{LH} / \mathrm{U} \cdot \mathrm{L}^{-1} \\
6 \mathrm{~m} \text { after operation }\end{array}$} \\
\hline Research & 50 & $17.31 \pm 1.57$ & $17.36 \pm 1.91$ & $18.45 \pm 1.73^{\triangle}$ & $20.15 \pm 1.43^{\triangle} *$ & $23.18 \pm 1.73^{\triangle} *$ \\
\hline Control & 50 & $18.47 \pm 1.94$ & $18.32 \pm 1.62$ & $22.03 \pm 1.87^{\triangle}$ & $23.19 \pm 1.78^{\triangle}$ & $27.15 \pm 1.69^{\triangle}$ \\
\hline \multirow{2}{*}{ Group } & \multirow{2}{*}{$\mathrm{n}$} & \multicolumn{5}{|c|}{$\mathrm{E} 2 /\left(\mathrm{pg} \cdot \mathrm{mL}^{-1}\right)$} \\
\hline & & before & $3 \mathrm{~m}$ after operation & 6 mafter operation & 1y after operation & $2 \mathrm{y}$ after operation \\
\hline Research & 50 & $346.12 \pm 16.42$ & $337.21 \pm 13.45^{*}$ & $321.04 \pm 10.36^{*}$ & $312.15 \pm 12.47^{* \Delta}$ & $298.34 \pm 12.08^{*^{\Delta}}$ \\
\hline Control & 50 & $343.24 \pm 17.64$ & $320.07 \pm 12.37^{\triangle}$ & $297.34 \pm 15.49^{\Delta}$ & $256.48 \pm 11.67^{\triangleright}$ & $203.17 \pm 12.09^{\triangle}$ \\
\hline
\end{tabular}

${ }^{\square} \mathrm{P}<0.05$ vs group compared in the previous observation time; ${ }^{\square} \mathrm{P}<0.05$ vs Control Compared in the same observation time.

\subsection{Comparison of incidence of peri-menopausal symptoms in the two groups}

Follow-up team followed up 50 cases, control group 50 cases; Two groups of postoperative incidence of perimenopausal symptoms is shown in table 3 .

Table 3. The comparison between incidence of peri-menopausal symptoms in the two groups

\begin{tabular}{|c|c|c|c|c|c|c|}
\hline Group & $\mathrm{n}$ & $\begin{array}{c}\text { Hot and } \\
\text { sweating(\%) }\end{array}$ & $\begin{array}{c}\text { palpitation } \\
(\%)\end{array}$ & $\begin{array}{c}\text { Insomia and } \\
\text { weak(\%) }\end{array}$ & $\begin{array}{c}\text { Loss of } \\
\text { libido(\%) }\end{array}$ & $\begin{array}{c}\text { Genital } \\
\text { atrophy(\%) }\end{array}$ \\
\hline Research & 50 & $2(4)$ & $2(4)$ & $5(10)$ & $4(8)$ & $1(2)$ \\
\hline Control & 50 & $15(30)$ & $12(24)$ & $26(52)$ & $23(46)$ & $6(12)$ \\
\hline $\mathrm{P}$ & & $<0.05$ & $<0.05$ & $<0.01$ & $<0.01$ & $>0.05$ \\
\hline
\end{tabular}




\section{Discussion}

The uterus is not only the target organ of ovarian hormone, but also has a close connection with the ovary in anatomical and endocrine function, both accept double blood supply. Uterine blood supply comes from the uterus branch of the uterine and ovarian arteries; Ovarian blood supply comes from the ovarian branch of the ovary and uterus artery. The literature reported that blood supply of the ovarian branch of the uterine artery accounted for $50 \% \sim 70 \%$ of ovarian blood supply[2]. Hysterectomy cut off the bilateral ovarian blood supply for preserved uterine arteries and veins, resulting in follicle degeneration, reduced hormone production, and the ovarian function declined after 4 5 years of operation[3]. The sub-total hysterectomy to maintain uterus blood vessels retained its normal blood supply of residual ovary, so ovarian secretion is not affected by the operation which can delay premature ovarian functiona aging, at the same time, the remaining uterus can secrete hormones, cytokines and maintain the endocrine balance between uterine and ovarian[4], maintain the normal function of the ovaries and play a role.

Siddle N[5] found that ovarian failure occured four years earlier than that of natural menopause after hysterectomy, ovarian failure rate is $34 \%$ with two years, increased to $54 \%$ within four years, the incidence of severe menopausal symptoms were significantly higher than the normal population. The experiment results showed that the serum follicle stimulating hormone (FSH) levels increased ,but estradiol (E2) levels droped in 50 patients for total hysterectomy compared with the control group during preoperative and postoperative periods, and perimenopausal symptoms were obvious; The amplitude change in serum sex hormone levels was less than the control group for 50 cases of subtotal hysterectomy maintaining uterine blood vessels during postoperative and preoperative times, and the incidence of perimenopausal symptoms were significantly lower than the control group.Research results showed that the ovarian branch of retaining uterine artery and vein affect ovarian blood supply less, reduced the effects of operation on ovarian hormone secretion; At the same time, there were no significant differences $(\mathrm{P}>0.05)$ in operation time, intraoperative blood loss, postoperative fever and postoperative residual polyp compared with the control group, which illustrated that uterus Hysterectomy of preservation uterine vessels did not increase the operation time and blood loss, also did not increase the postoperative infection and postoperative complications.

A number of studies have confirmed that estrogen has a protective effect on cardiovascular system, the low level of serum sex hormone levels and atherosclerosis is associated with high cardiovascular events [6]. Research has shown that decreased estrogen levels cause atherosclerosis, elevated blood lipids can enhance incidence of coronary heart disease by tow-folds, which constitued one of the main risk factors of the coronary heart disease(CHD). E2 decrease of normal postmenopausal women cause blood lipid disorders, dyslipidemia is the important cause of the occurrence of coronary heart disease in postmenopausal women; Similarly, E2 levels drop can also lead to blood lipid disorders in premenopausal women after hysterectomy[6]. This study result showed that there was no obvious changes in hormone levels in serum for retension of uterus blood vessels of sub-total hysterectomy group, FSH increased significantly in traditional sub-total hysterectomy. Study results suggested the traditional total hysterectomy retained the ovaries, but reduced ovarian blood supply caused by cutting off uterine arteriovenous branches, affected ovarian endocrine function, droped E2 levels, which may affect blood lipid level, its effect on blood lipid specific remained to be confirmed by next step research); Reserved with uterine arteriovenous ovarian surgery can reduce the influence on ovarian blood supply, reduced the influence on ovarian hormone secretion, there were no significant decline in serum E2 level in Subtotal hysterectomy for maintaining uterine vessels (STHMUV) compared with the preoperative group, meanwhile reduced incidence of perimenopausal symptoms. people have higher requirement of life quality with the progress of the society. we should choose hysterectomy that has smaller influence on ovarian function for the non-menopause women with gynecological benign diseases, retain ovarian branch of uterine arteriovenous supply as far as possible, which can reduce the effect on ovarian hormone secretion, slowed the emergence of ovarian premature aging, and at the same time also can reduce the incidence of postoperative coronary heart disease; the operation is 
simple,safe,easy to master,less postoperative complications, all these provide a good surgical option $[7-10]$.

\section{References}

1. Xu Xiaofeng, Zhou Huandi, Li Miao. J.Nanchang Univer, 50,18 (2010)

2. Wu Fengying, Deng Zhaohong, Tian song. Xinjiang med, 39,86 (2009)

3. Cicinelli E,Einer-Jensen N, Galantino P.. Ann NY Acad Sci,1034,19 (2004)

4. Chen Lu, Li Guangyi, Han Yubin. Chinese J of mini-trauma surgery, 9, 609 (2009)

5. Siddle N,Sarrel P,Whitehead H..Fertil Steril,,47,94 (1987)

6. Mohamad M J,Karayyem M,Mohammad M A,et al.,Am J Phys Anthropol,,132,151(2007)

7. Li Kaijun, $\mathrm{Wu}$ CongCong ,Xiang Yijia,et al. prevention and treatment of cardiovascular and cerebrovascular disease, 14,222 (2014)

8. Wang Jingjing, He Ruhua, Wu Ling. Ningxia med J,36,13 (2014)

9. Sheng Li, Ye Ping. Chin J of prac Int Med, 34,15 (2014)

10. Gimbel H..Acta Obstet Gynecol Scand,86, 133 (2007) 